\title{
The development of early attachment: An observation study on an infant throughout the first year
}

\author{
Gu Chuan Hua ${ }^{1}$ \\ School of Psychology, Huazhong Normal University, Wu Han
}

\begin{abstract}
Since 1950s, the development of early attachment has been paid great attention to. However until now few longitudinal studies continuing for a long time were conducted on the process of attachment development. Moreover little attention was paid to the mechanism of attachment emergence and the relationship between after-birth attachment and prenatal environment although researchers put more emphasis on the development of after-birth attachment of children. In the case study, Sunshine, a healthy female infant was observed naturally for one year to investigate deeply the micromechanism of early attachment of children and put forward the related hypotheses.

Sunshine was born on the fourteenth of February, 2006, with normal DQ (developmental quotient) and easy type (Thomas et al., 1977) and uninhibitant type (Kagan, 1987) of temperament, and she was observed naturally by a professional developmentalist within one year. In the process of observation all kinds of her typical behavior including eating, drinking, sleep, bathing, social interaction and action, were registered objectively every day.

The results indicated that different stages of attachment development existed, and the close relationship of attachment development and prenatal environment was obviously found. Sunshine developed from the first stage of "uterus attachment"(from birth to about 1.5 months old) to the second stage of "uterus-like attachment"(about 1.56 months old), including generalized "uterus-like attachment"(1.5-2.5 months old) and differentiated "uterus-like attachment" (2.5-6 months old), and then to the third stage of "social uterus attachment"(6 months after birth), and with each stage characterized by the correspondent typical behavioral mode. And the developmental process of early attachment is essentially characterized by the gradual promotion of socialization extent.

The earliest "uterus-like attachment" reflected her attachment to the living and nurturing environment. Maternal body or uterus was a kind of enwrapped environment with enough security, warmth, comfort, stability, appropriate moving and with the automatic provision of enough oxygen and all kinds of nutrition, and natural and convenient physical dissimilation process. However after-birth environment asked her to independently obtain nutrition, feeling of warmth, feeling of comfort and security, which easily caused her attachment to maternal uterus, and the uterus-like environment more easily bring about her preference and remembrance of maternal uterus.

On the stage of generalized "uterus-like attachment"(1.5-2.5 months old), the attachment behavior could be aroused by the environment like uterus or the environment which could arouse the feeling like the feeling of the uterus. And on the stage of differentiated "uterus-like attachment" (2.5-6 months old), the attachment behavior could be aroused by the stable environment which was like uterus and which was provided by familiar adults, and these adults might become figures of her
\end{abstract}

\footnotetext{
${ }^{1}$ School of Psychology, Central China Normal University, 430079, China. Address: Hongshan District of Wuhan City No. 100 Luo Yu Road, 430079. E-mail: guchuanhua_19740701@tom.com.
} 
attachment probably. On the third stage of "social uterus attachment"( 6 months after birth), Sunshine could form the attachment relationship with familiar adults who could created the "social uterus" which could provide enough stability, certainty, security and could aroused the feeling of control over environment through the adults' appropriate nurturing behavior. In the environment she could predict the change of environment, and the familiar adults might become the concrete figures of her attachment. The feeling like that of uterus might be caused by the social interaction of Sunshine with familiar caregiver. The "social uterus attachment" might be the attachment to the stable environment which could be predicted in nature.

The research provided new understanding of early attachment of children, and redefined the starting point of attachment. The following hypotheses can be proposed that, (1) "uterus attachment" is the starting point of children's attachment, and afterbirth attachment can be seen as the extension and continuation of "uterus attachment" in social environment, and the deep relationship may existed between the two. (2) emotional warmth and security provided by adults may be the determinant factors of children's attachment quality. 
早期依恋的发展 : 对一个婴儿第一年行为的观察研究

谷传华 ${ }^{2}$

（华中师范大学心理学院，武汉，430079）

摘要: 为深入探讨儿童依恋发生、发展的微观机制, 由一名专业的发展心理学研究 者采用自然观察法, 在一年内（2006.4.14-

2007.4.14）每天对一名健康女婴Sunshine（化名）进行客观记录或登记。结果显示, Suns hine的依恋经历了一个由“子宫依恋”（0-1.5个月左右最明显）到“类子宫依恋”（1.56个月左右最典型）, 再到 “社会子宫依恋”（6个月以后明显）的发展过程, 其中 “类 子宫依恋”阶段又分为泛化的“类子宫依恋”阶段（1.5-

2.5 个月左右最典型）与分化的“类子宫依恋”阶段（约2.5-

6 个月最典型），每个阶段都有相应的典型行为模式。依恋的发展实质上是一个社会化程 度不断提高的过程。

\section{1. 引言}

自从20世纪六、七十年代以来, 早期依恋的发展一直得到研究者的高度关注, 以J. Bowlby, M.D.S.

Ainsworth等人为代表的心理学家, 对依恋的发展、影响因素、研究方法以及干预等开展 了一系列理论和实证研究, 奠定了依恋研究的基础 (Bretherton, 1992; Howe,

2006)。例如，R. Schaffer等人(Schaffer \& Emerson,

1964)研究发现，早期儿童的依恋可划分为非社会性阶段、未分化的依恋阶段、分化的依 恋阶段、多重依恋阶段四个阶段 ; 类似地, Newman等人(Newman \& Newman， 2005)则总结了Bowlby(1969, 1982), Answorth(1973, 1985),

Marvin和Britner(1999)等人的研究, 把依恋的发展分为 0 3

个月（没有依恋反应）、3 6个月（对熟人和生人的反应分化）、6 9个月（依恋关系开

${ }^{2}$ 作者单位: 华中师范大学心理学院, 430079 - 通讯地址 : 武汉市洪山区珞喻路100号, 430079. Email: guchuanhua 19740701@tom.com 
始建立）、9 12个月（依恋关系明确化）、12个月以后（依恋关系增强，与依恋对象形 成目标协调的伙伴关系）等多个阶段。但是，迄今关于儿童早期依恋发展的结论主要是 根据对婴儿大样本的追踪研究或实验研究得出来的, 在这些研究中, 研究者或者对婴儿 的父母进行定期访谈, 或者对婴儿在陌生情境中的行为进行观察, 而对儿童进行长期深 入的追踪观察, 考察早期依恋发展过程的研究并不多。而且, 研究者一般偏重儿童出生 后依恋关系建立的过程, 尤其是依恋关系建立后的行为, 而对依恋发生发展的深层微观 机制、产后依恋与孕期环境的关系等问题，很少给予关注。

本研究对一名典型儿童从出生后进行了为期一年的自然观察, 试图深入探讨儿童早 期的依恋发生、发展的微观机制, 在此基础上提出关于儿童发生、发展机制的研究假设

\section{2. 研究对象}

Sunshine（化名）, 一名女婴, 2006年4月14日出生, 剖腹产, 出生体重为3.45千克 , 用Apgar量表(Apgar,1974), 从心率、呼吸、肌肉弹性、皮肤颜色、反射敏感性五个方 面进行评价，约得10分，即满分，说明Sunshine出生时身体健康状况良好。

Sunshine智能发育正常。采用首都儿科研究所（1988）在丹佛发育篮查测验（DDST ）和韦氏学龄前儿童智力测验量表(WPPSI)基础上编制的0～6岁小儿神经心理发育量表， 从认知能力、精细运动能力、大肌肉运动能力、语言能力、社交能力（包括自理能力） 5 个方面，对婴儿的发育状况进行逐月测查，发现其发育商均在90110之间。另外，根据Thomas等人(1977)与Kagan(1987)的气质理论（Papalia， 2005），可以把Sunshine划入容易型、非抑制型气质。

\section{3. 研究过程}

采用自然观察法, 由一名专业的发展心理学研究者 (教育学博士) 为观察者, 对她 每天的行为, 包括饮食、睡眠、洗澡、排泄、社会性行为和反应进行详细记录或登记。 在最初6个月，对每天发生的所有行为进行全程记录 ; 在随后的半年，对全天发生的典型 
行为或典型事件进行记录或登记。观察记录避免了观察者个人成见、情绪的影响, 力求 客观、真实，观察内容举例见文后。

\section{4. 研究结果与分析}

观察结果显示, Sunshine出生后依恋的发展与孕期的发育环境具有密切的关系, 发 展过程表现出明显的阶段性。根据其出生后在各种典型情境中的行为及其与环境之间的 互动特点，可以把Sunshine依恋的发展过程具体划分为以下几个阶段。

\section{1 依恋的发生：母体依恋或子宫依恋（0-1.5个月左右最明显）}

Sunshine的依恋在出生后就已发生，最初的依恋主要是对母体子宫环境的依恋，大 约在出生后的1个半月内最为明显。这表现于其出生后各种典型的生活情境中。

\subsection{1 饮食}

在饮食情境中，下列典型行为可以清楚地反映Sunshine对母亲子宫环境的依恋。

（1）吃奶成为最重要的生活内容：在哭闹时，喂奶可以很快地使她平静下来，但在 母乳不充足时喂母乳, 则哭泣不停, 在出生后的最初几天即如此。一方面, 充足的奶水 可以消除饥饿感，另一方面，由奶嘴吸食充足的奶水类似于在子宫中吞咽羊水的情形。

（2）喜欢在母亲怀抱中吃奶: 出生后5天, 只要放在母亲一侧或母亲一抱起她, 她 就转身寻找乳头，做吃奶状; 在成人怀抱中将要入睡时，也总是转身张口、伸舌，闭着 眼做寻找奶头吃奶状。与母亲相依偎可以带来类似于子宫环境中的感受。

（3）含乳头与奶嘴入睡 : 在烦躁不能入睡时, 含乳头或奶嘴容易入睡, 而且, 在拿 掉奶嘴时则容易苏醒。乳头或奶嘴可带来足够的类似于子宫羊水般的充实感和安全感。 
4.1.2 睡眠

在睡眠情境中，下列典型行为或反应可以清楚地反映她的子宫依恋倾向。

（1）自然的睡眠姿势：仰卧，两臂侧上举，大臂平举，小臂与大臂成90 度角, 双腿向外自然弯曲, 这与子宫中的自然弯曲基本一致。

（2）睡眠方式：喜欢在成人怀抱中入睡，哭闹时抱起则平静，放在床上则容易哭闹 ，十分喜欢摇动，尤其喜欢让成人抱着慢慢地走动或抖动，轻轻地拍打身体。

（3）摇篮: 在摇篮中容易入睡, 苏醒之后, 轻轻地、反复地摇动摇篮, 可以重新入 睡。这类似于胎儿生活的子宫环境，母体的运动可能促成了追求这种动感的倾向。摇篮 也是中外母亲抚育和安慰婴儿时最常用的床具, 它反映了婴儿的某种普遍的倾向或需要

（4）睡眠环境：夜间在光线较弱的环境中容易入睡，在明亮的环境中容易苏醒，不 容易入睡。暗光环境更类似于母体的子宫环境。

（5）哼唱：睡眠时成人反复地、轻轻地哼唱同样的小曲，或者念叨同样的话语或单 词, 更容易入睡; 如果烦躁不安, 播放舒缓轻柔的摇篮曲, 就能很快安静下来, 并逐渐 进入睡眠。轻柔的有规律的声音或乐音类似于子宫环境中的声音刺激, 特别是母亲血液 流动的声音以及内脏活动的声音。

4.1.3 洗澡

特别喜欢洗澡, 在身体有支撑的情况下尤其如此, 最初成人让她抓住手指, 就能安 静地坐在浴盆的温水中洗澡, 接受成人的洗浴; 在室温为 $29 \circ \mathrm{C}-$ 30 ㄷ 的环境中, 洗澡水的温度在 $36 \circ C-40$ ㄷ

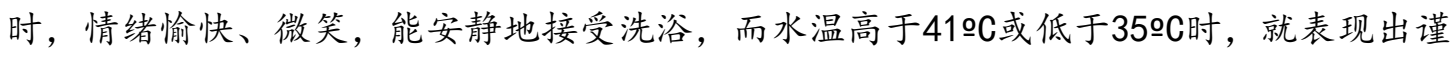
慎、紧张或肌肉收缩、闭眼睛等反应, 甚至大声哭闹, 拒绝洗澡。在澡盆中身体寻找的 支撑与子宫内身体得到的支撑相似，这种水温与正常的人体温度或子宫内环境的温度类 似，而这种温度下的水环境也类似于子宫内的羊水环境。 
在本质上，这种母体依恋或子宫依恋是她对自身生存和发育环境的一种单向的依恋 。母体的子宫环境是一种“包裹式”的环境，羊水可以使胎儿免受母体震动的影响，保持 适宜的环境温度, 并能使胎儿在一种无重力的环境中自由移动; 从子宫内层和线毛膜发 育而成胎盘则为胎儿提供氧气和养料、排泄胎儿的代谢废物。因此, 在通常情况下, 子 宫舒适、安全、温暖、稳定, 动静适宜, 氧气和各种养分的供给自动、及时而充足, 排 泄或生理异化过程安适而自然。出生后的环境则要求婴儿独立地获取营养、温暖感、安 全感、舒适感, 因而势必要与成人进行积极的互动, 通过肢体的活动、声音、表情等手 段，向成人表明自身生理与心理的状态、需要。这种由环境变化导致的从“自动”到“主动 ”的转变，很容易促成她对子宫环境的依恋，在身体尚未充分适应外界环境、尚缺乏独立 生存能力的情况下，“回归”子宫的倾向尤其明显，因而类似于子宫内环境的环境也更容 易引起婴儿的偏爱和对子宫的“怀念”。

\section{2 依恋的发展：从“类子宫依恋”到“社会子宫依恋”}

Sunshine的依恋经历了一个由“子宫依恋”到“类子宫依恋”，再到“社会子宫依 恋” 的发展过程。

\section{2.1 由泛化的“类子宫依恋”到分化的“类子宫依恋”（1.5-6个月左右最典型）}

在这一时期, 能够满足婴儿营养需要, 为婴儿创造一种类似于子宫的抚养环境的成 人，更容易成为婴儿出生后依恋的对象，引起贞儿的“类子宫依恋”。

最初是泛化的 “类子宫依恋” ，Sunshine对任何成人所创造的类似于子宫环境的环 境都会偏爱, 而对这类环境的创造者也会表现出依恋倾向, 而无论是谁, 即依恋对象尚 未分化 ; 后来，逐渐发展为分化的 “类子宫依恋”，对于稳定地创造 “类子宫环境” 的 成人或熟人，表现出分化的、更明显的依恋倾向。

（1）泛化的“类子宫依恋”（1.5-2.5个月左右最典型）

在这一阶段, 无论成人具有怎样的特点, 只要能够创造类似于子宫, 或能引起类似 于子宫感受的环境, 就能够引起Sunshine的偏爱或依恋。由于动态的人能够创造这类环 
境, 而静态的事物本身并不能提供这类环境, 因而人成为这一阶段Sunshine关注的对象 ，对人的活动更为敏感，对人的反应也更多、更积极。这具体表现为以下典型行为：

在苏醒状态下，对人声敏感，如果有人经过旁边或听到说话声，她就会转头看，甚 至停止吃奶, 注视; 家人与陌生人在温和地抱起她的时候, 她都能注视人们的面孔, 安 静、不哭闹; 熟人不温和的态度和行为也会引起不愉快的反应, 如果家人比较严厉, 对 她的哭闹感到厌烦，态度、行为和言语不温和（如厉声呵斥她），她就停顿片刻，看着 家人 (父亲) 的面孔, 随后继续大哭不止, 如果家人转为温和地抱起她, 轻轻地拍打、 抚慰，她就会停止哭闹，并很快入睡; 如果陌生人面部表情严肃，抱她或接近她，她就 会注视着陌生人, 或者扭过脸去, 显得比较谨慎, 如果陌生人温和地抱她, 对她笑, 她 也会微笑、高兴起来。

（2）分化的“类子宫依恋”（约2.5-6个月最为典型）

在这一阶段, 熟悉的成人创造的相对稳定的、不确定感较小的、类似于子宫的环境 , 才能引起Sunshine更多的偏爱, 因此, 熟悉的成人更可能成为他们的依恋对象, 陌生 人即使能创造这样一种环境, 但因其短暂性、不稳定性、不确定性, 短期内也难以引起 真正的偏爱或依恋。这种日益分化的依恋，与Sunshine日益接受熟悉的成人的 “子宫式 ” 的抚养模式有关, Sunshine有可能形成一种内部工作模式, 并开始作为衡量别的社会 环境或刺激是否可靠的标准。这具体表现为以下典型行为。

对家人表现出更多的积极反应: 在吃饱、家庭气氛温暖的情况下, 如果家人对她表 现的表情、行为温和, 说话的声音轻柔、舒缓, 或者在洗澡时、愉快地逗她时, 她都会 显得十分愉快, 看着成人的面孔微笑; 喜欢与自己年龄相仿的婴儿: 见到娃娃, 显得很 高兴, 如果娃娃友好地对她笑, 她也能对着娃娃微笑, 显得很愉快; 非常喜欢看卡通电 视节目, 刚开始看的时候, 就对节目中友好的卡通宝宝表现出浓厚的兴趣和高度集中的 注意力, 有时兴奋地叫起来（婴儿的形态和声音可能会带来更多的安全感和温暖感）; 对陌生人的亲热反应减少: 家中来陌生人的时候, 她表现出越来越多的谨慎, 对爸爸、 妈妈和外婆的微笑、亲近反应显著多于陌生人，对陌生人盯着面孔看，甚至长时间地盯 着看，不笑，但对陌生人仍然有友好反应。

4. 2.2 由分化的 “类子宫依恋” 到 “社会子宫依恋”（6个月以后明显） 
这一阶段, Sunshine能够与熟悉的成人形成具体的依恋关系, 熟悉的抚养者通过各 种适当的抚养行为, 为婴儿创造稳定的、确定性的、可以预测和控制的环境氛围, 即能 创造一种社会性的 “子宫” , 使㚣儿能够在其中获得足够的安全感、稳定感、确定感以 及可预测、可控制感, 因而他们更可能成为婴儿具体的依恋对象。这种类似于子宫环境 的感受是婴儿通过与熟悉的抚养者积极的社会互动促成的。社会子宫依恋实质上是对可 预测的、稳定的动态环境的依恋。这具体表现为以下典型行为：

怯生更明显, 陌生人到家中, 如果父亲在场, 抱着她, 她会看着陌生人怯生生地微 笑; 如果爸爸不在场, 陌生人抱着, 就没有明显的反应, 即使陌生人逗引她也不笑, 甚 至会哭起来, 不看陌生人; 与生人与家人的反应明显分化, 陌生人来家里, 抱起她, 父 亲装作离开她的样子, 看着爸爸离开, 焦虑不安, 想哭, 不看生人, 昂昂地叫, 父亲回 来, 笑起来, 让父亲抱; 父亲在抱着她时, 陌生人拍手要抱, 她扭头躲避; 她每天都忍 受着与父母亲分离的苦恼, 每当父母出门离开她的时候, 她都感到极大的烦恼: 哭泣, 如果有人抱着, 她就会侧身向着父母的方向挣扎, 追随着他们, 发出啊啊、昂昂的声音 , 好像对他们离开自己表示反抗, 父母每次回来后则微笑、高兴, 让父母抱。

显然, 经过了半年左右的时间, 她已经由一种单向的强烈的子宫依恋倾向, 发展为 类子宫依恋和随后的社会子宫依恋, 而到12个月结束时, 这种社会子宫依恋倾向变得更 为明显而典型。这一时期, Sunshine已经产生了明显的陌生人谨慎(caution of strangers)和分离焦虑(anxiety of separation), 按照Ainsworth等人(Ainsworth, Blehar, Waters, \& Wall, 1987; Bretherton, 1990)的依恋类型划分标准和上述表现, 她属于典型的安全型依恋(Newman \& Newman, 2005)。

\section{5. 结论和讨论}

通过以上分析, 不难得出以下结论 :

（1）Sunshine的依恋在出生后即已产生, 最初的依恋主要表现为她对母体子宫环境 的依恋，即子宫依恋或母体依恋； 
（2）Sunshine依恋的发展经历了一个由“子宫依恋”到“类子宫依恋”，再到“社 会子宫依恋”的发展过程，其中类子宫依恋又分为泛化的“类子宫依恋”（1.52.5 个月左右最典型）与分化的“类子宫依恋”（2.56个月左右最典型），6个月以后逐渐发展为社会子宫依恋；

（3）Sunshine依恋的发生与孕期的母体环境或子宫环境具有密切的关系，依恋的发 展实质上是一个社会化程度不断提高的过程。

把依恋划分为若干发展阶段并不意味着，依恋的发展是连续发生跳跃性质变的过程 ，恰恰相反，依恋发展的过程同时具有连续性与阶段性，而连续性的变化是阶段性变化 的基础，阶段性的变化体现着更大程度的连续性变化，阶段之间是连续的，不能跨越一 个阶段而直接跃入另一个阶段。

如果进行一个比较, 我们就会发现, Sunshine的依恋行为模式发生转折性变化的时 间点与众多研究的结论基本一致。例如，在时间上，本研究中的子宫依恋阶段基本对应 于H. R. Schaffer等人(Schaffer \& Emerson, 1964)的第1阶段一非社会性阶段（0-

1.5个月），而分化的类子宫依恋阶段基本对应于其第2阶段一未分化的依恋阶段（1.57个月）与Bowlby(1969, 1982)等人的分化反应阶段（3-

6个月），而社会子宫依恋分别相当于他们的分化依恋阶段和依恋关系建立、明确期。这 在一定程度上说明, 婴儿的依恋具有某种相对稳定的发展趋势, 它具有跨文化的一致性

本研究的意义不在于它验证了这一发展趋势, 而在于它为我们理解依恋产生和发展 的机制提供了一个新的视角，并重新定义了依恋发展的起点。以往有关的研究描绘了依 恋发展的趋势以及早期依恋与后来（包括成年后）依恋的连续性(McDonald, et al., 2005)，而不能充分地解释最初依恋发展的原因，没有深入分析孕期的母体环境与产后的 社会环境、个体在母体环境中的心理和行为倾向与产后依恋行为的关系，事实上，我们 无法否认和割裂两种环境、两种行为倾向之间的联系或连续性。从这个角度出发, 我们 就不难理解Answorth等人(1987)所划分的不同依恋类型的儿童的各种行为以及抚养质量对 早期依恋发展的影响(Ainsworth, 1979; De Wolff \& van ljzendoorn, 1997), 不难理解H. F. Harlow(1958)研究中的幼猴更亲近可提供温暖的木制母猴而不是铁丝母猴的原因，同时也 不难理解S. Freud所谓的口唇期（0-1岁）的儿童行为以及C.

Rogers所谓的 “无条件积极关注” 的意义。正如本研究结果所表明的那样，儿童的依恋 本质上更可能是对可提供足够安全感、温暖感的生存或生活环境的依恋，这种依恋在出 生之后就已表现出来，随后会逐渐转化为对能够提供或创设特定环境的人的依恋。 
从上述分析, 我们可以提出以下假设：

（1）母体依恋或子宫依恋是个体依恋的起点；

（2）出生后的依恋是子宫依恋在社会环境中的自然延续或延伸，两类依恋的发展具 有连续性和深层次的联系;

（3）成人提供的情感温暖和安全感是决定儿童依恋质量的核心因素。

近年来, 不断变化的社会条件（包括出生方式、母亲就业等）对父母养育行为和早 期儿童发展的影响日益引起人们的关注, 成为本世纪发展心理领域新的研究方向(Fiske, et al., 2004)。成长环境对依恋发展的影响也得到高度关注(Caldera \& Lindsey, 2006;

李同归，2006)。例如，大量研究一致表明，父母的敏感性和反应性是影响早期儿童依恋 安全性的基本因素之一, 即使对于那些有心理障碍的儿童也是如此(van IJzendoorn, et al., 2007; Barnett, et al., 2006; Juffer, et al., 2005)。本研究对象的父母敏感性、反应性较高, 因而其依恋的安全性程度较高。在此意 义上, 本研究的结论可能代表了父母敏感性、反应性较高的安全型依恋的儿童的发展过 程，而在父母敏感性、反应性很低的家庭环境中成长的儿童可能具有不同的发展模式。 而且，气质、文化等因素也可能会影响儿童依恋的特点(Shaffer, 2004; 张文新， 1999)。因此，我们有必要把上述假设放到文化背景、气质、出生方式和家庭环境不同的 婴儿群体中进行验证, 以得到更为确切的结论。

\section{参考文献}

Answorth, M.D.S. (1973). The development of infant-mother attachment. In B. M. Caldwell \& H. N. Ricciuti (Eds.), Review of child development research (Vol. 3). Chicago: University of Chicago Press

Ainsworth, M.D.S. (1979). Infant-mother attachment. American Psychologist, 34, 932-937

Answorth, M.D.S. (1985). Patterns of infant-mother attachments: Antecedents and effects on development. Bulletin of the New York Academy of Medicine, 61, 771-791

Ainsworth, M.D.S, Blehar, M.C., Waters, E., \& Wall, S. (1987). Patterns of attachment: A psychological study of the strange situation. Hillsdale, NJ: Erlbaum.

Barnett, D., Clements, M., Kaplan-Estrin, M., McCaskill, J.W., Hill Hunt, K., Butler, C.M., Schram, J.L., \& Janisse, H.C. (2006). Maternal resolution of child diagnosis: stability 
and relations with child attachment across the toddler to preschooler transition. Journal of Family Psychology, 20(1), 100-107.

Bowlby, J. (1969/1982). Attachment and loss: Attachment (Vol. 1). New York: Basic Books.

Bretherton, I. (1992). The origins of attachment theory: John Bowlby and Mary Ainsworth. Developmental Psychology, 28, 759-775.

Caldera, Y.M., Lindsey, E.W. (2006). Coparenting, Mother-Infant Interaction, and InfantParent Attachment Relationships in Two-Parent Families. Journal of Family Psychology, 20(2), 275-283.

Fiske, S.T., Schacter, D.L., Zahn-Waxler, C. (2004). Annual Review of Psychology, 55, 385399.

Harlow, H.F. (1958). The nature of love. American Psychologist, 13, 673-685.

Hock, R.R. (2004). Forty studies that changed psychology: Explorations into the history of psychological research (pp. 167-177). New Jersey: Prentice Hall.

Howe, D. (2006). Developmental attachment psychotherapy with fostered and adopted children. Child and Adolescent Mental Health, 11(3), 128-134.

Juffer, F., Bakermans-Kranenburg, M.J., van IJzendoorn, M.H. (2005). The importance of parenting in the development of disorganized attachment: evidence from a preventive intervention study in adoptive families. Journal of Child Psychology and Psychiatry, 46(3), 263-274.

Marvin, RS, \& Britner, P. A. (1999). Normative development: The ontogeny of attachment. In J. Cassidy \& P. R. Shaver (Eds.), Handbook of attachment: Theory, research, and clinical applications (pp. 44-67). New York: Guilford Press.

McDonald, A., Beach, P., Beck, R., Allison, S., \& Norsworthy, L. (2005). Attachment to God and Parents: Testing the Correspondence vs. Compensation Hypotheses. Journal of Psychology and Christianity, 24(1), 21-28,

Newman, B.M., \& Newman, E.R.. (2005). Development through life: A psychosocial approach. America: Wadsworth Press (Chinese edition).

Papalia, D.E., Olds, S.W., Feldman, \& R.D. (2005). Human Development (9th edition). New York: McGraw-Hill. 
Schaffer, H.R., \& Emerson, P.E. (1964). The development of social attachments in infancy. Monographs of the Society for Research in Child Development, 29(3, Serial no. 94).

Shaffer, D.R. (2004). Developmental Psychology: Childhood and Adolescence (sixth edition). Thomson Learning and China Light Industry Press.

Van IJzendoorn, M.H., Rutgers, A.H., Bakermans-Kranenburg, M.J., et al. (2007). Parental sensitivity and attachment in children with autism spectrum disorder: comparison with children with mental retardation, with language delays, and with typical development. Child Development, 78(2): 597-609.

李同归. 母亲的依恋类型与教养方式的相关性研究. 中国行为医学科学, 2006, 15, 149-150 张文新. 儿童社会性发展. 北京 : 北京师范大学出版社，1999. 210-219

附：观察记录举例（片段）-一

9月17日（上半年）

$11: 00$

困倦, 烦躁, 爸爸抱着走动, 渐渐入睡, 放在床上, 立即醒来, 重新抱起走动, 入睡。

$12: 30$

醒, 自己听音乐, 玩玩具, 不哭闹, 对着玩具咿呀 “说话”; 爸爸喂奶粉和水, 不拒绝 ; 手中某个玩具或东西掉在地上，咿呀 “说话”，看爸爸的脸，好像想让爸爸帮忙拾起 来; 扶着沙发或柱子站起来，很兴奋，叫，看爸爸的脸；爸爸把尿，不哭闹，如果不想 尿，就挺直身体，抬头看爸爸的脸，像在说 “我不想”。

爸爸抱起走动, 渐渐困倦闭眼, 但刚放在床上, 马上醒来, 不睁眼哭闹; 爸爸继续抱着 走动、渐渐入睡时，侧身做吮奶的样子，将手指放在嘴里。

\section{1月4日（下半年）}

早晨醒来后，自己静静地看周围，不哭闹，见到爸爸、妈妈，情绪愉快，微笑，在休息 充足之后，都表现出类似的反应。

上午, 妈妈抱她出去, 旁边的老人要抱她, 她拒绝; 而当老人抱着她的时候, 妈妈拍手 ，伸出双臂，急切地让妈妈抱。 
中午, 自己拿着一个小夕子, 重复地掷在地板或沙发上, 爸爸为她捡起多次, 都是如此 , 当她掊在地上时, 爸爸故意发出惊奇的声音, 她还为自己的行为笑出声来。

下午, 爸爸抱着她, 大伯来, 要抱抱她, 拒绝, 将头扭过去, 想哭, 不让抱, 过了一阵 , 她才让他抱, 但显得极为谨慎, 已经表现出对陌生人的谨慎或怯生。

晚上, 爸爸在身边, 她自己扶着小车后背站起来, 腾出一手去抓电话; 在她探索的过程 中, 不时会看看身边的爸爸, 当她站着用手打开小车顶蓬的时候, 看爸爸一眼, 自己笑 出声来; 睡觉前, 妈妈、爸爸为她洗澡, 十分高兴, 坐在水中, 两手抓住盆沿, 看水, 用两腿、两脚蹬水, 后来用一只手抓住盆沿, 另一只手不断地拍水, 当把她从水中抱出 的时候, 包括穿衣服时, 她都坚决拒绝, 不愿从水中出来, 反抗, 大声哭闹, 而在唱歌 哄她的时候才逐渐安静下来。 